\title{
State Dependent NGMV Control of Delayed Piecewise Affine Systems
}

\author{
Yan Pang and M. J. Grimble, IEEE Fellow
}

\begin{abstract}
A Nonlinear Generalized Minimum Variance (NGMV) control algorithm is introduced for the control of delayed piecewise affine (PWA) systems which are an important subclass of hybrid systems. Under some conditions, discrete-time PWA systems can be transferred into their equivalent state dependent nonlinear systems form. The equivalent state dependent systems that include reference and disturbances models are very general. The process is assumed to include common delays in input or output channels of magnitude $k$. Then the NGMV control strategy [16] can be applied. The NGMV controller is related to a well-known and accepted solution for time delay systems but has the advantage that it can stabilize open-loop unstable processes [17].
\end{abstract}

\section{INTRODUCTION}

In the past several years, methodologies have been developed to model hybrid systems, to analyze their behavior, and to synthesize controllers that guarantee closed-loop stability and performance specifications. Researchers have become increasingly interested in the framework related to the stability and the control problems for a specific class of systems called piecewise affine ( $P W A$ ) systems.

This growth in interest is partly motivated by the fact that many nonlinear systems can be approximated (arbitrarily closely) using piecewise affine systems [1] and because the interconnection of finite automata and linear systems yields piecewise affine system descriptions [2]. In fact, piecewise-affine systems represent a broad modeling class in the sense that they have been shown to be equivalent to many other classes of systems, such as mixed logic dynamical (MLD) systems [3] and extended linear complementary systems [4]. PWA systems therefore represent a possible starting point in the study of both hybrid and nonlinear systems.

Control algorithms developed for piecewise affine systems are often designed using optimal control or Model Predictive Control (MPC) techniques. The first hybrid MPC algorithm, developed for mixed logical dynamical systems (equivalent to piecewise affine systems under certain mild conditions), was presented in [3]. Unfortunately, this algorithm has a drawback that it has a high on-line computational demand. This is mainly caused by the mixed integer quadratic programming problem ( $N P$ hard) that has to be solved on-line, at each discrete-time instant.

The delayed discrete-time PWA model in this paper is more general than most PWA models. It includes not only disturbances but also time delays. Therefore, many existing

The authors are with the Industrial Control Centre, University of Strathclyde, 50 George Street, Glasgow G1 1QE, UK (e-mail: yan.pang@strath.ac.uk,m.grimble@eee.strath.ac.uk). control strategies are not effective for this type of model. Motivated by the fact that state dependent systems are much easier to be controlled and estimated as both the state and input constraints and the switching conditions for state dependent systems can be included in the system model. Thus an equivalent state dependent framework for PWA systems is developed under some assumed conditions.

State dependent systems also arise when parametric uncertainty is present in a model [5] or when the actual nonlinear system can be approximated by a state dependent system and an LTI model is a poor approximation. The advantages of this approach are:

1. State dependent model needs less supervision by logical constructs than controllers developed with traditional techniques for hybrid systems.

2. System time delay and disturbances are more naturally modeled in the plant than many other existing hybrid control system models (eg. $M L D$ ).

3. It is easy to extend to systems with other types of nonlinearities or uncertainties.

After obtaining the state dependent model, the so called Nonlinear Generalized Minimum Variance (NGMV) controller, which is very simple to compute and implement, can be applied. In the following the process of obtaining the model and properties of the control law are explored. The focus is on implementation and design issues.

\section{SYSTEM DESCRIPTION}

\section{A. PWA Systems}

In this work, we focus on delayed discrete-time PWA systems, whose state-space representation is:

$$
\begin{array}{r}
x(t+1)=A_{i} x(t)+B_{i} u(t-k)+D_{i} d(t) \\
y(t)=C_{i} x(t)+E_{i} u(t-k)
\end{array}
$$

where $x \in \mathbb{R}^{n}$ is the state, $u \in \mathbb{R}^{m}$ is the input, $y \in \mathbb{R}^{p}$ is the output and $d \in \mathbb{R}^{n}$ is the disturbance. $k$ denotes the magnitude of the common delay elements. Each affine subsystem $\left(A_{i}, B_{i}, C_{i}, D_{i}, E_{i}\right), i=1, \ldots, s$ is defined on a cell $\Omega_{i} \subset \mathbb{R}^{n} \times \mathbb{R}^{m}$ that is a polyhedron. Moreover, in order to simplify the exposition, we assume that our cells are polyhedral sets defined by matrices $G_{i x}, h_{i x} G_{i u}$ and $h_{i u}$

$$
\left.\Omega_{1}=\left\{\begin{array}{l}
x \\
u
\end{array}\right] \mid G_{i x} x \leq h_{i x} \wedge G_{u} u \leq h_{u}\right\}
$$

The cells satisfy $\Omega_{\mathrm{i}} \cap \Omega_{\mathrm{j}}=\phi, \forall \mathrm{i} \neq \mathrm{j}$, their union defines the admissible set of states and inputs $\Omega=\bigcup_{i=1}^{S} \Omega_{i}$. 
Although PWA systems have been studied in many papers, the system disturbances $(d(t)$ in system (1)) are not included in most of them, for example [7]. For those existing works focused on piecewise affine systems with disturbances [8], disturbances can only belong to a small bounded set. Unlike above models, the disturbances in this work are assumed to be zero-mean, independent, Gaussian white noise. Another complicated part of this model is that it includes the common delay $\mathrm{k}$ which is also rare in the existing literatures.

A PWA system (1) is called well-posed [9], if $x(t+1), y(\mathrm{t})$ are uniquely defined functions of $x(t), u(t-k), d(t)$. For a well-posed PWA system, the sets $\Omega_{\mathrm{i}}$ have mutually disjoint interiors, and are often defined as the partition of a convex polyhedral set. i.e. $\Omega_{\mathrm{i}} \cap \Omega_{\mathrm{j}}=\phi, \forall \mathrm{i} \neq \mathrm{j}$.

Note that the well-posedness requirement of a PWA system is contradicted with the definition in equation (2), where $\Omega_{\mathrm{i}}$ and $\Omega_{\mathrm{j}}$ can have overlapping boundaries from the definition " $\leq$ ". To ensure the well-posedness, some of the inequalities in equation (2) can be written in $G_{i x} x<h_{i x}$ and $G_{i u} u<h_{i u}$ forms.

\section{B. State Dependent Systems}

A state dependent system involves state equation matrices that are time-varying depending upon the states also upon control input:

$$
\begin{aligned}
& x(t+1)=\mathcal{A}(x, u) x(t)+\mathcal{B}(x, u) u(t-k)+\mathcal{D}(x, u) d(t) \\
& y(t)=\mathcal{C}(x, u) x(t)+\mathcal{E}(x, u) u(t-k)
\end{aligned}
$$

The state dependent formulism is simple, systematic model that is applicable to a wide range of nonlinear dynamical systems. It can express evolutions of continuous (linear) variables through linear dynamic equations, of discrete (nonlinear) variables through propositional logic statements, and the mutual interaction between the two. State dependent systems are therefore capable of modeling a broad class of systems, in particular those systems that can be modeled through PWA systems.

Proposition 1: Every well-posed PWA system can be written as a state-dependent system i.e. for any feasible polyhedral partition of state + input set $\Omega=\cup_{i=1}^{S} \Omega_{i}$ and its corresponding parameters $\left(A_{i}, B_{i}, C_{i}, D_{i}, E_{i}\right), \mathrm{i}=1, \ldots, \mathrm{s}$, of system (1), there exists a combination of $(\mathcal{A}(x, u), \mathcal{B}(x, u), \mathcal{C}(x, u), \mathcal{D}(x, u), \mathcal{E}(x, u))$ of system (3), such that all the trajectories $x(t), u(t), y(t)$ of the PWA system (1) also satisfy the state dependent (3).

Proof: Consider the PWA system (1), to rephrase the condition (2) in logic form, we introduce an auxiliary logic variable $\delta_{i}(t) \in\{0,1\}$, where:

$$
\delta_{i}(t)=\left\{\begin{array}{cc}
1 \quad \text { if } G_{i x} x(t) \leq h_{i x} \wedge G_{i u} u(t) \leq h_{i u} \\
0 \quad \text { otherwise }
\end{array}\right.
$$

A well- posed system (1) with the partition (2), then can be written in the following form:

$$
\begin{aligned}
& x(t+1)=\sum_{i=1}^{s} \delta_{i}(t)\left[A_{i} x(t)+B_{i} u(t-k)+D_{i} d(t)\right] \\
& y(t)=\sum_{i=1}^{s} \delta_{i}(t)\left[C_{i} x(t)+E_{i} u(t-k)\right]
\end{aligned}
$$

The value of logic variable $\delta_{i}(t) \in\{0,1\}$ in system (4) depends on the state and input variables $x(t)$ and $u(t)$. Defining the less than or equal $(\leq)$ function $\mathrm{LE}(\mathrm{x}, \mathrm{m})$ as:

$$
L E(x, m)= \begin{cases}1 & \text { if } x \leq m \\ 0 & \text { otherwise }\end{cases}
$$

where $\mathrm{m}$ is a scalar. Therefore

$$
\delta_{i}(t)=\prod_{j} L E\left(G_{i x}^{j} x(t), h_{i x}^{j}\right) \prod_{l} L E\left(G_{i u}^{l} u(t), h_{i u}^{l}\right)
$$

where $j$ and 1 denote the $j$-th row and the l-th row respectively. By substituting (5) in (4a) and (4b) we obtain:

$$
\begin{aligned}
x(t+1)= & {\left[\sum_{i=1}^{s} \prod_{j} L E\left(G_{i x}^{j} x(t), h_{i x}^{j}\right) \prod_{l} L E\left(G_{i u}^{l} u(t), h_{i u}^{l}\right) A_{i}\right] x(t)+} \\
& {\left[\sum_{i=1}^{s} \prod_{j} L E\left(G_{i x}^{j} x(t), h_{i x}^{j}\right) \prod_{l} L E\left(G_{i u}^{l} u(t), h_{i u}^{l}\right) B_{i}\right] u(t-k)+} \\
& {\left[\sum_{i=1}^{s} \prod_{j} L E\left(G_{i x}^{j} x(t), h_{i x}^{j}\right) \prod_{l} L E\left(G_{i u}^{l} u(t), h_{i u}^{l}\right) D_{i}\right] d(t) }
\end{aligned}
$$$$
\begin{aligned}
y(t)= & {\left[\sum_{i=1}^{s} \prod_{j} L E\left(G_{i x}^{j} x(t), h_{i x}^{j}\right) \prod_{l} L E\left(G_{i u}^{l} u(t), h_{i u}^{l}\right) C_{i}\right] x(t)+} \\
& {\left[\sum_{i=1}^{s} \prod_{j} L E\left(G_{i x}^{j} x(t), h_{i x}^{j}\right) \prod_{l} L E\left(G_{i u}^{l} u(t), h_{i u}^{l}\right) E_{i}\right] u(t-k) }
\end{aligned}
$$

Hence, the PWA system (1) is transformed into a non-linear state-dependent system (6) which has the formulism of system (3) where

$$
\begin{aligned}
& \mathcal{A}(x, u)=\sum_{i=1}^{s} \prod_{j} L E\left(G_{i x}^{j} x(t), h_{i x}^{j}\right) \prod_{l} L E\left(G_{i u}^{l} u(t), h_{i u}^{l}\right) A_{i} \\
& \mathcal{B}(x, u)=\sum_{i=1}^{s} \prod_{j} L E\left(G_{i x}^{j} x(t), h_{i x}^{j}\right) \prod_{l} L E\left(G_{i u}^{l} u(t), h_{i u}^{l}\right) B_{i} \\
& \mathcal{C}(x, u)=\sum_{i=1}^{s} \prod_{j} L E\left(G_{i x}^{j} x(t), h_{i x}^{j}\right) \prod_{l} L E\left(G_{i u}^{l} u(t), h_{i u}^{l}\right) C_{i} \\
& \mathcal{D}(x, u)=\sum_{i=1}^{s} \prod_{j} L E\left(G_{i x}^{j} x(t), h_{i x}^{j}\right) \prod_{l} L E\left(G_{i u}^{l} u(t), h_{i u}^{l}\right) D_{i}
\end{aligned}
$$

and

$$
\mathcal{E}(x, u)=\sum_{i=1}^{s} \prod_{j} L E\left(G_{i x}^{j} x(t), h_{i x}^{j}\right) \prod_{l} L E\left(G_{i u}^{l} u(t), h_{i u}^{l}\right) E_{i}
$$

Note that the well-posedness of original PWA system implies that $\delta_{i}(t)$ and $L E(\bullet, \bullet)$ are $\{0,1\}$-valued, and 
$\sum_{i=1}^{s} \delta_{i}=1 . \quad$ In general, the feasible state + input set $\Omega$ of

(2) is non-convex, i.e. there must be some inequalities take the ' $<$ ' form. Nevertheless, the ' $<$ ' function can also be defined like the ' $\leq$ ' function as:

$$
L T(x, m)= \begin{cases}1 & \text { if } x<m \\ 0 & \text { otherwise }\end{cases}
$$

Both $L E(x, m)$ and $L T(x, m)$ can be calculated using Matlab function LE.m and LT.m respectively.

From Proposition 1, an equivalent state dependent system can always be found for a well-posed PWA system. The advantage of state-dependent systems over PWA systems is that state-dependent systems are much easier to be controlled and estimated as both the state and input constraints and the switching conditions are all included in the system model. Hence, the Nonlinear Generalized Minimum Variance (NGMV) control of state-dependent systems will be introduced in the next section.

\section{SYSTEM MODEL DESCRIPTION}

\section{A. System Plants}

In order to derive the control algorithm for state dependent systems, we use the general system description in [6]. The plant is nonlinear and may include two nonlinear subsystems. Considering the input signals are normally bounded for PWA systems, the first nonlinear subsystem is defined as a saturation nonlinear system in this paper. The second is a so called state-dependent non-linear equation form. However, the reference and disturbance signals are assumed to have linear model representations. The system is shown in Fig.1, including the nonlinear plant model and the linear reference/disturbance models. The zero mean white measurement noise is denoted $\{v(t)\}$ and it has a covariance matrix $R_{f}$. There is no loss of generality in assuming that the zero-mean, white noise signals $\{\omega(t)\}$ and $\left\{\xi_{2}(t)\right\}$, that feed the reference and disturbance models, have identity covariance matrices.

The state-space linear and non-linear system models and the non-linear operator model [11], [12] may be listed as follows:

\section{Reference model:}

$x_{r}(t+1)=A_{r} x_{r}(t)+D_{r} \omega(t), \quad x_{r}(t) \in R^{n_{r}}$

$r(t)=C_{r} x_{r}(t)$ and $W_{r}\left(z^{-1}\right)=C_{r}\left(z I-A_{r}\right)^{-1} D_{r}$

\section{Error weighting:}

$$
\begin{aligned}
& x_{p}(t+1)=A_{p} x_{p}(t)+B_{p}\left(r(t)-y_{2}(t)\right), x_{p}(t) \in R^{n_{p}} \\
& y_{p}(t)=C_{p} x_{p}(t)+E_{p}\left(r(t)-y_{2}(t)\right)
\end{aligned}
$$

Nonlinear Plant: $(\mathcal{W} u)(t)=z^{-k}\left(\mathcal{W}_{k} u\right)(t)$

The total forward path plant model:

$$
(\mathcal{W u})(\mathrm{t})=\mathrm{z}^{-\mathrm{k}}\left(\mathcal{W}_{2 \mathrm{k}} \mathcal{W}_{1 \mathrm{k}} \mathrm{u}\right)(\mathrm{t})
$$

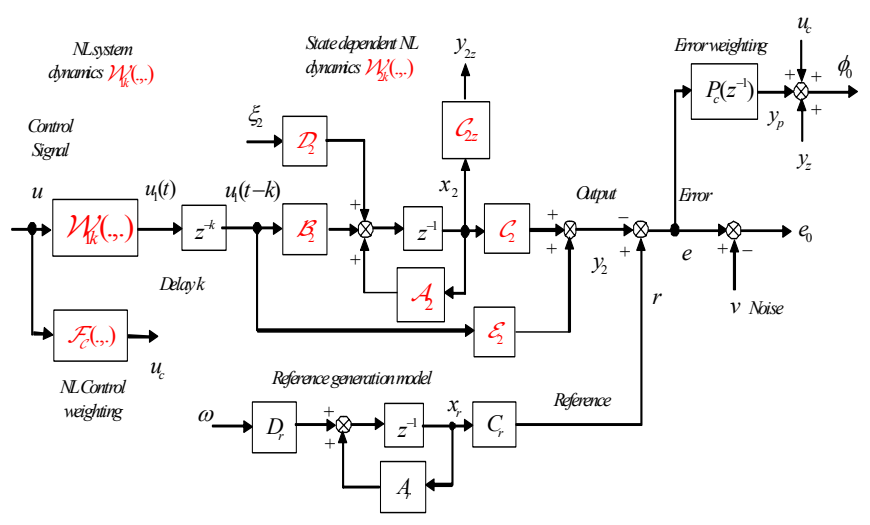

Fig.1. State-Dependent Plant Model Including Linear Disturbance and Reference Models and $u_{1}(t)=\mathcal{W}_{1 k}(.,) u.(t)$. Although the first nonlinear subsystem can be a general nonlinear system [6], a saturation nonlinear system is defined here to insure the input signal $u_{1}(t)$ is bounded i.e. $u_{1}(t) \in\left[u_{\min }, u_{\max }\right]$ where $u_{\min }$ and $u_{\max }$ are the lower and upper bound. The second nonlinear subsystem is represented by the state-dependent model equations, shown in Fig.1.

Total Linear Sub-System State Equation Model: Combining the linear reference and error weighting model, obtain the augmented state equations for the total linear sub-system as:

$$
\begin{aligned}
& x(t+1)=A x(t)+B y_{2}(t)+D \xi(t) \\
& y(t)=C x(t)+E y_{2}(t)
\end{aligned}
$$

where the system state vector $x(t)=\left[x_{r}{ }^{T}, x_{p}^{T}\right]^{T}$. The resolvent operator may now be defined as: $\Phi\left(z^{-1}\right)=(z I-A)^{-1}$ so that,

$$
x(t)=\Phi\left(z^{-1}\right) B y_{2}(t)+\Phi\left(z^{-1}\right) D \xi(t)
$$

\section{$B$ State Prediction Equations}

The Kalman filter is needed to estimate the states of the combined linear model. These results are well known [10] and will be omitted here. Define:

$$
\begin{aligned}
& T_{0}\left(k, z^{-1}\right)=\left(I-A^{k} z^{-k}\right) \Phi\left(z^{-1}\right)= \\
& z^{-1}\left(I+z^{-1} A+z^{-2} A^{2}+\ldots+z^{-k+1} A^{k-1}\right)
\end{aligned}
$$

which denotes a transfer operator with finite pulse response. A $k$-step ahead state prediction can be expressed as

$$
\begin{aligned}
& \hat{x}(t+k \mid t)=A^{k} \hat{x}(t \mid t)+T_{0}\left(k, z^{-1}\right) B y_{2}(t+k) \\
& =A^{k} \hat{x}(t \mid t)+A^{k-1} B y_{2}(t)+A^{k-2} B y_{2}(t+1)+\ldots \ldots \\
& \quad+A B y_{2}(t+k-2)+B y_{2}(t+k-1)
\end{aligned}
$$

Now consider the second nonlinear system model in the so-called linear state-dependent (LSD) state-space form [13]. It is the system defined in (3) with $\mathrm{k}$ steps common delay:

$$
\begin{aligned}
& x_{2}(t+1)=\mathcal{A}_{2}\left(x_{2}, u_{1}\right) x_{2}(t)+\mathcal{B}_{2}\left(x_{2}, u_{1}\right) u_{1}(t-k) \\
& +\mathcal{D}_{2}\left(x_{2}, u_{1}\right) \xi_{2}(t) \\
& y_{2}(t)=\mathcal{C}_{2}\left(x_{2}, u_{1}\right) x_{2}(t)+\mathcal{E}_{2} u_{1}(t-k)
\end{aligned}
$$

where $x_{2}(\mathrm{t})$ is a vector of sub-system states, $u_{1}(t)$ is a vector of the LSD sub-system inputs, $y_{2}(t)$ is a vector of sub-system 
output signals and $\xi_{2}(t)$ is a vector of disturbance signals, assumed to be zero-mean, independent, Gaussian white noise. To simplify notation in (17), write $\mathcal{A}_{2}(t)=\mathcal{A}_{2}\left(x_{2}(t), u_{1}(t)\right)$ and similarly for $\mathcal{B}_{2}, \mathcal{C}_{2}, \mathcal{D}_{2}$ and $\mathcal{E}_{2}$.

Prediction model: The k-steps prediction of the state and output signals will therefore be defined,

$$
\begin{gathered}
\mathcal{T}_{1}\left(k, z^{-1}\right)=\mathcal{A}_{2}(t+k-1) \mathcal{A}_{2}(t+k-2) \ldots \mathcal{A}_{2}(t+1) \mathcal{B}_{2}(t) z^{-k} \\
+\ldots+\mathcal{A}_{2}(t+k-1) \mathcal{B}_{2}(t+k-2) z^{-2}+\mathcal{B}_{2}(t+k-1) z^{-1} \\
\multicolumn{2}{c}{\hat{x}_{2}(t+k \mid t)=\mathcal{A}_{2}(t+k-1) \mathcal{A}_{2}(t+k-2) \ldots} \\
\mathcal{A}_{2}(t) \hat{x}_{2}(t \mid t)+\mathcal{T}_{1}\left(k, z^{-1}\right) u_{1}(t) \\
(19) \\
\hat{y}_{2}(t+k \mid t)=\mathcal{C}_{2}(t+k) \mathcal{A}_{2}(t+k-1) \mathcal{A}_{2}(t+k-2) \ldots \\
\mathcal{A}_{2}(t) \hat{x}_{2}(t \mid t)+\left(\mathcal{C}_{2}(t+k) \mathcal{T}_{1}\left(k, z^{-1}\right)+\mathcal{E}_{2}(t+k)\right) u_{1}(t)
\end{gathered}
$$

\section{Nonlinear GMV CONTROL LAW}

\section{A . NGMV Control Problem}

The signal $\left\{\phi_{0}(t)\right\}$ is to be minimized in a variance sense, where:

$$
\phi_{0}(t)=P_{c} e(t)+\left(\mathcal{Z}_{c} x\right)(t)+\left(\mathcal{F}_{c} u\right)(t)
$$

and the cost index to be minimized:

$$
J=E\left\{\phi_{0}^{T}(t) \phi_{0}(t)\right\}=E\left\{\operatorname{trace}\left\{\phi_{0}(t) \phi_{0}^{T}(t)\right\}\right\}
$$

where $E\{\cdot\}$ denotes the unconditional expectation operator.

The signal $\left\{\phi_{0}(t)\right\}$ involves an error signal dynamic cost function weighting matrix $P_{c}\left(z^{-1}\right)$, that is represented by a linear state-space sub-system with output: $y_{p}(t)=P_{c} e(t)$ where $e(t)=r(t)-y(t)$ is the error signal. A weighting on a certain combination of states may be included in the criterion via the signal:

$$
y_{z}(t)=\left(\mathcal{Z}_{c} \mathcal{X}\right)(t)=C_{1 z} x(t)+\mathcal{C}_{2 z} x_{2}(t), \text { where }
$$

$y_{1 z}(t)=\mathcal{C}_{1 z} x(t)$ and $y_{2 z}(t)=\mathcal{C}_{2 z} x_{2}(t)$.The operator:

$\mathcal{Z}_{c}$ can include dynamics and nonlinear terms, and for simplicity can be augmented to the second LSD sub-system states.

The control signal weighting can be defined to have the form: $\quad\left(\mathcal{F}_{\mathrm{c}} u\right)(t)=z^{-k}\left(\mathcal{F}_{\mathrm{c} k} u\right)(t)$

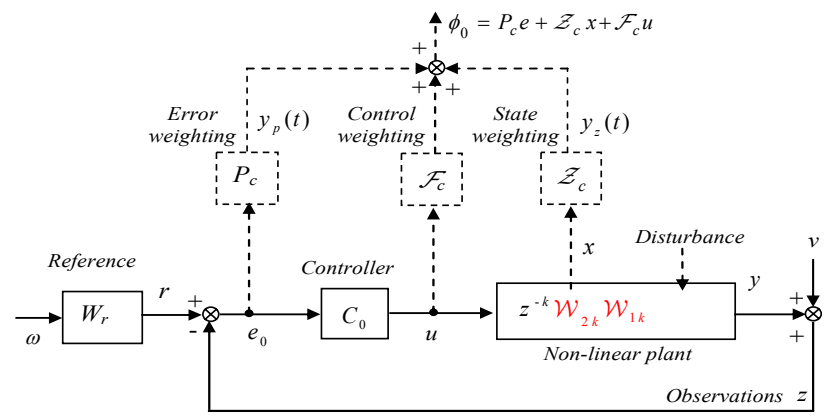

Fig. 2: Single Degree of Freedom Closed-Loop Control System for the Nonlinear Plant
B. Solution of the NGMV Optimal Control Problem

The solution of the optimal control problem may be obtained by expanding the expression for the inferred output $\left\{\phi_{0}(t)\right\}$ and by then introducing a prediction equation.

Recall,

$\phi_{0}(t)=P_{c} e(t)+\left(\mathcal{Z}_{c} \mathcal{X}\right)(t)+\left(\mathcal{F}_{c} u\right)(t)=y_{p}(t)+y_{z}(t)+\left(\mathcal{F}_{c} u\right)(t)$

The first error weighting term may be written in a more concise form, using (17), as:

$$
y_{p}(t)=P_{c} e(t)=C_{1 p} x(t)+E_{1 p} y_{2}(t)
$$

where $x(t) \in R^{n}, E_{1 p}=-E_{p}$. The state weighting term:

$y_{z}(t)=\left(\mathcal{Z}_{c} \mathcal{X}\right)(t)=C_{1 z} x(t)+\mathcal{C}_{2 z} x_{2}(t)$

Thence, the inferred output or signal to be minimized:

$$
\phi_{0}(t)=\mathcal{C}_{\phi} \mathcal{X}(t)+E_{\phi} u_{0}(t-k)+\left(\mathcal{F}_{c} u\right)(t)
$$

where $\mathcal{C}_{\phi}=\left[\begin{array}{ll}C_{\phi 1} & \mathcal{C}_{\phi 2}\end{array}\right]=\left[\begin{array}{ll}\left(C_{1 p}+C_{1 z}\right) & \mathcal{C}_{2 z}\end{array}\right]$ and $E_{\phi}=E_{1 p}$. So that,

$\phi_{0}(t)=\mathcal{C}_{\phi} \mathcal{X}(t)+E_{\phi}\left(\mathcal{W}_{2 k} \mathcal{W}_{1 k} u\right)(t-k)+\left(\mathcal{F}_{c} u\right)(t)$

In the set of channels with explicit delay $k$ the control signal affects the outputs $\phi_{0}(t)$ at least $k$ steps later and the control signal weighting was therefore defined to have the form: $\left(\mathcal{F}_{c} u\right)(t)=z^{-k}\left(\mathcal{F}_{c k} u\right)(t)$. Substituting into equation (25) obtain:

$$
\phi_{0}(t)=\mathcal{C}_{\phi} \mathcal{X}(t)+\left(\left(E_{\phi} \mathcal{W}_{2 k} \mathcal{W}_{1 k}^{(32)} \mathcal{F}_{c k}\right) u\right)(t-k)
$$

The prediction may be obtained in terms of (15) and (19) as:

$$
\begin{aligned}
& \hat{\mathcal{X}}(t+k \mid t)=\left[\begin{array}{c}
\hat{x}(t+k \mid t) \\
\hat{x}_{2}(t+k \mid t)
\end{array}\right] \\
& =\left[\begin{array}{c}
A^{k} \hat{x}(t \mid t)+T_{0}\left(k, z^{-1}\right) B y_{2}(t+k) \\
\mathcal{A}_{2}(t+k-1) \mathcal{A}_{2}(t+k-2) \ldots \mathcal{A}_{2}(t) \hat{x}_{2}(t \mid t)+\mathcal{T}_{1}\left(k, z^{-1}\right) u_{1}(t)
\end{array}\right] \\
& =\left[\begin{array}{cc}
A^{k} & 0 \\
0 & \mathcal{A}_{2}(t+k-1) \mathcal{A}_{2}(t+k-2) \ldots \mathcal{A}_{2}(t)
\end{array}\right]\left[\begin{array}{c}
\hat{x}(t \mid t) \\
\hat{x}_{2}(t \mid t)
\end{array}\right] \\
& +\left[\begin{array}{c}
T_{0}\left(k, z^{-1}\right) B W_{2 k} \\
\mathcal{T}_{1}\left(k, z^{-1}\right)
\end{array}\right] u_{1}(t)
\end{aligned}
$$

which may be written more concisely, with an obvious definition of matrix terms, as:

$$
\hat{\mathcal{X}}(t+k \mid t)=\mathcal{A}_{\phi}^{k} \hat{\mathcal{X}}(t \mid t)+\mathcal{T}_{\phi}\left(k, z^{-1}\right) u_{1}(t)
$$

Note that the predicted values of the state related terms in (24) therefore become:

$$
\begin{aligned}
& \mathcal{C}_{\phi} \hat{\mathcal{X}}(t+k \mid t)=C_{\phi 1} A^{k} \hat{x}(t \mid t)+\mathcal{C}_{\phi 2} \mathcal{A}_{2}(t+k-1) \mathcal{A}_{2}(t+k-2) \\
& \ldots \mathcal{A}_{2}(t) \hat{x}_{2}(t \mid t)+\left(C_{\phi 1} T_{0}\left(k, z^{-1}\right) B \mathcal{W}_{2 k}+\mathcal{C}_{\phi 2} \mathcal{T}_{1}\left(k, z^{-1}\right)\right) u_{1}(t)
\end{aligned}
$$

Prediction Equation: The $k$ steps ahead prediction of the signal: $\phi_{0}(t)$, from (26) and (27), may now be obtained as:

$$
\begin{aligned}
& \hat{\phi}_{0}(t+k \mid t)=\mathcal{C}_{\phi} \hat{\mathcal{X}}(t+k \mid t)+\left(\left(E_{\phi} \mathcal{W}_{2 k} \mathcal{W}_{1 k}+\mathcal{F}_{c k}\right) u\right)(t) \\
& =\mathcal{C}_{\phi} \mathcal{A}_{\phi}^{k} \hat{\mathcal{X}}(t \mid t)+\left(\left(\left(\mathcal{C}_{\phi} \mathcal{T}_{\phi}\left(k, z^{-1}\right)+E_{\phi} \mathcal{W}_{2 k}\right) \mathcal{W}_{1 k}+\mathcal{F}_{c k}\right) u\right)(t)
\end{aligned}
$$

The cost-function involves the minimization of the weighted error and control signals, in a variance sense. The variance: $J=E\left\{\phi_{0}(t+k)^{T} \phi_{0}(t+k)\right\}$ may be written in 
terms of the prediction $\hat{\phi}_{0}(\mathrm{t}+\mathrm{k} \mid \mathrm{t})$ and the prediction error: $\tilde{\phi}_{0}(t+k \mid t)$, using the orthogonality properties [14], as:

$$
J=\mathrm{E}\left\{\phi_{0}(t+k \mid t)^{T} \hat{\phi}_{0}(t+k \mid t)\right\}+\mathrm{E}\left\{\tilde{\phi}_{0}(t+k \mid t)^{T} \tilde{\phi}_{0}(t+k \mid t)\right\}
$$

Theorem 1: NGMV Optimal Controller for State Dependent and NL Systems

The NGMV optimal controller to minimize the variance of the weighted error, states and control signals may be computed from the following state and operator equations. The assumption is made that the nonlinear operator: $\left(\mathrm{P}_{\mathrm{c}} \mathcal{W}_{\mathrm{k}}-\mathcal{N}_{\mathrm{c}}-\mathcal{F}_{\mathrm{ck}}\right)$ has a finite gain $m_{2}$ stable causal inverse, due to the choice of weighting operators $P_{c}, \mathcal{Z}_{c}$ and $\mathcal{F}_{c k}$.

Optimal control signal: The optimal NGMV control action can be computed as:

$$
\begin{aligned}
& \mathrm{u}(\mathrm{t})=-\left(\mathcal{F}_{\mathrm{ck}}+\left(\mathcal{C}_{\phi} \mathcal{T}_{\phi}\left(\mathrm{k}, \mathrm{z}^{-1}\right)+\mathrm{E}_{\phi} \mathcal{W}_{2 \mathrm{k}}\right) \mathcal{W}_{\mathrm{lk}}\right)^{-1} \mathcal{C}_{\phi} \mathcal{A}_{\phi}^{\mathrm{k}} \hat{\mathcal{X}}(\mathrm{t} \mid \mathrm{t}) \\
& \text { or } \\
& \mathrm{u}(\mathrm{t})=-\mathcal{F}_{\mathrm{ck}}^{-1}\left(\mathcal{C}_{\phi} \mathcal{A}_{\phi}^{\mathrm{k}} \hat{\mathcal{X}}(\mathrm{t} \mid \mathrm{t})+\left(\mathcal{C}_{\phi} \mathcal{T}_{\phi}\left(\mathrm{k}, \mathrm{z}^{-1}\right)+\mathrm{E}_{\phi} \mathcal{W}_{2 \mathrm{k}}\right)\left(\mathcal{W}_{\mathrm{lk}} \mathrm{u}\right)(\mathrm{t} \mathrm{t})\right.
\end{aligned}
$$

where $E_{\phi}=-E_{p}$.

\section{Application to Vehicle traction Control}

Traction controllers are used to improve a driver's ability to control a vehicle under adverse external conditions such as wet or icy roads. The objective of the controller is to maximize the traction force while preserving stability. The traction force depends on adhesion coefficient between the tire and road surface, which in turn depends on the wheel slip as well as the tire/road surface condition.

The overall control scheme is composed of two parts: a device that estimates the road surface condition and a traction controller that regulates the wheel slip at desired values. Since the paper focuses on NGMV control for PWA systems, we assume that an exact estimate of road friction is available that means we only focus on the second part of the control scheme.

\section{A. Dynamics Model for Traction Control}

Following the formulation proposed in [15], a simple model is used for the design of the traction controller. The simple mechanical system is shown in Fig. 3.

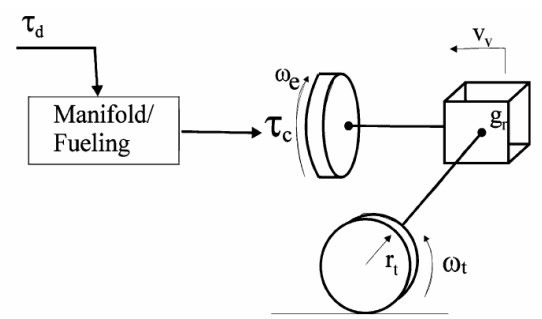

Fig. 3. A simple vehicle model
In [15], the friction torque $\tau_{t}$ is approximated as a piecewise linear function and a PWA system (32) is obtained by discretizing the system model:

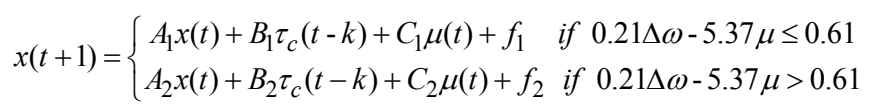

$y(t)=\Delta \omega(t)$

where $x=\left[\begin{array}{ll}\omega_{e} & v_{v}\end{array}\right]^{T}, \omega_{e}$ is the engine speed and $v_{v}$ is the vehicle speed. $\mathrm{k}$ denotes the time delay between the actual combustion torque $\tau_{c}$ and the desired combustion torque $\tau_{d}$, $\mu$ denotes road coefficient of friction and the slip $\Delta \omega$ is defined as the difference between the normalized vehicle and engine speeds. The parameters are:

$A_{1}=\left[\begin{array}{cc}0.98316 & 0.78486 \\ 0.00023134 & 0.989220\end{array}\right], B_{1}=\left[\begin{array}{c}0.048368 \\ 5.6688 e-6\end{array}\right], C_{1}=\left[\begin{array}{c}-0.35415 \\ 0.0048655\end{array}\right], f_{1}=\left[\begin{array}{c}0.10934 \\ -0.0015034\end{array}\right]$

$A_{2}=\left[\begin{array}{cc}1.0005 & -0.021835 \\ 6.4359 e-6 & -1.00030\end{array}\right], B_{2}=\left[\begin{array}{c}0.048792 \\ -1.5695 e-7\end{array}\right], C_{2}=\left[\begin{array}{c}-6.5287 \\ 0.089695\end{array}\right], f_{2}=\left[\begin{array}{c}0.081687 \\ -0.011223\end{array}\right]$

\section{B. Nonlinear State Dependent Model}

Several important characteristics of the traction control problem have made the NGMV control of the state-dependent model (PWA) very convenient and effective:

- The nonlinear frictional torque $\tau_{t}$ has a clear piecewise linear behavior that can be transferred into a state dependent model very easily following Proposition 1 .

- The logical variables are embedded in the state dependent model; hence $N G M V$ controller requires much less supervision by logical constructs than controllers developed with traditional techniques (eg. PID)

- In general, the system's dynamics should include external disturbances. However, the $M L D$ model in [15] is not able to model the system disturbances so easily, whilst disturbances are modeled in state dependent systems (3) in a natural way.

- Unlike in [15] where the $\mathrm{k}$ steps time delay between $\tau_{c}$ and $\tau_{d}$ was taken into account only during implementation of the control law, it can also be modeled naturally by the state dependent model (17).

- The state dependent structure is easy to be extended to handle more accurate model eg. more concrete time varying uncertainties are modeled as a function of state or input without changing the model format.

Hence, for above reasons the friction torque $\tau_{t}$ can be modeled by following state dependent system: $x(t+1)=L E(0.21 \Delta \omega-5.37 \mu, 0.61) \cdot\left[A_{1} x(t)+B_{1} \tau_{c}(t-k)+C_{1} \mu(t)+\right.$ $\left.f_{1}\right]+G T(0.21 \Delta \omega-5.37 \mu, 0.61) \cdot\left[A_{2} x(t)+B_{2} \tau_{c}(t-k)+C_{2} \mu(t)+f_{2}\right]$

$y(t)=\Delta \omega(t)$

where $x=\left[\begin{array}{ll}\omega_{e} & v_{v}\end{array}\right]^{T}, \mathrm{k}$ denotes the time delay of $\tau_{c}$. 


\section{B. NGMV Controller Design}

In the control process the system receives the desired wheel slip $\Delta \omega_{d}$, the estimated road coefficient adhesion $\mu$, and the measured front and rear wheel speeds as input, then generates the engine torque $\tau_{c}$. The $N G M V$ controller is applied on a state dependent system (33) with the initial conditions of that $\mu=0.2$ (ice surface), $\omega_{e}(0)=180 \mathrm{rad} / \mathrm{s}$, $v_{v}=0 \mathrm{~m} / \mathrm{s}$, time delay $\mathrm{k}=3$, using the following parameters:

Reference model: $W_{r}\left(z^{-1}\right)=\frac{0.15 z^{-1}}{1-z^{-1}}$

Error weighting: $\quad P_{c}\left(z^{-1}\right)=\frac{440-785 z^{-1}+376.2 z^{-2}}{1-1.5 z^{-1}+0.5 z^{-2}}$

Control weighting: $F_{c k}=-1.5+0.3 z^{-1}$

The following constraints have to be satisfied: $-20 \mathrm{Nm} \leq \tau_{c} \leq 200 \mathrm{Nm}, \dot{\tau}_{c} \leq 2000 \mathrm{Nm} / \mathrm{s}$ and $\Delta \omega \geq 0$

The simulation results for a ramp and step references are shown in the following figures.

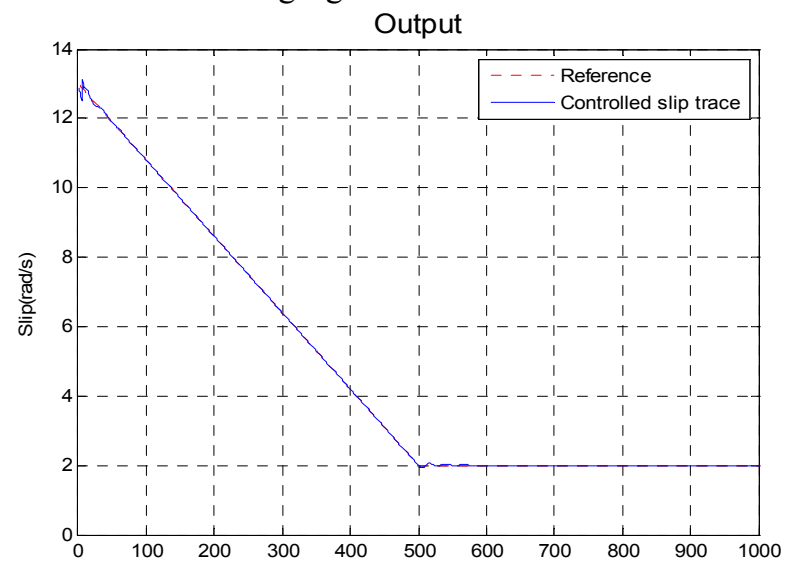

Fig 4: Simulation results of NGMV controller (Reference slip $\Delta \omega_{d}=2 \mathrm{rad} / \mathrm{s}$ )

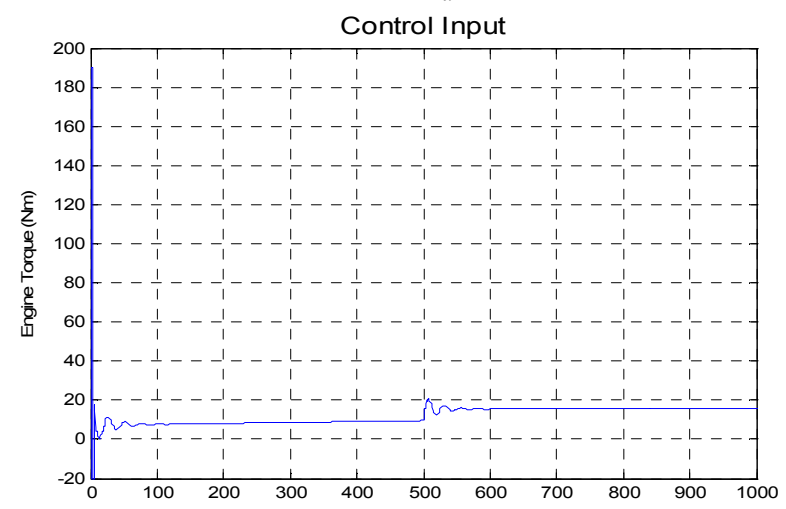

Fig 5: Engine torque command

It is clear in Fig 4 that the $N G M V$ controller has a very good performance in tracking the slip reference.

\section{CONCLUDING REMARKS}

An $N G M V$ controller for delayed PWA systems, whose switching sequence depends on the state and on the control input, has been proposed. These PWA systems can be translated into NL state-dependent systems by introducing some binary functions which are the conditions of crossing the switching surfaces. The advantage of state dependent systems over PWA systems is that state-dependent systems are easier to control and estimate as both the state and input constraints and the switching conditions are included in the system mode. The state-feedback NGMV design methodology provides an alternative way to synthesize controllers for hybrid systems. However, there are some hybrid systems where the switching conditions are more complicated that cannot be modeled as PWA systems. The state-dependent systems may be extended to model these types of hybrid system. A discrete supervisor is needed for this extension and an NGMV controller for the continuous control part.

\section{REFERENCES}

[1] Sontag. E D. Nonlinear regulation: the piecewise linear approach, IEEE Transactions on Automatic Control, 26(2), pp. 346-357, (1981).

[2] Sontag. E.D. Interconnected automata and linear systems: A theoretical framework in discrete-time, In Hybrid systems III, (R. Alur, T.A. Henzinger, and E.D. Sontag, Ed. Springer), pp. 436-448, (1996).

[3] Bemporad, A. and Morari, M., Control of systems integrating logic, dynamics, and constraints. Automatica, 35(3):407-427.(1999)

[4] Schutter, B. D. and Moor, B. D. The extended linear complementarity problem and the modeling and analysis of hybrid systems. In Antsaklis, P., Kohn, W., Lemmon, M., Nerode, A., and Sastry, S., editors, Hybrid Systems V: Verification and Control: Lecture Notes in Computer Science, Vol. 1567, pp. 70-85.Springer-Verlag.1999

[5] Blanchini. F. Ultimate boundedness control for uncertain discrete-time systems via set-induced Lyapunov functions, IEEE Trans. Aut. Control, 39(2):428-433, 1994.

[6] Grimble, M. J. State dependent nonlinear generalised minimum variance control of multivariable systems. Tech. report, Industrial Control Centre, University of Strathclyde. 2004

[7] Bemporad, A., Ferrari-Trecate, G. and Morari, M. Observability and Controllability of Piecewise Affine and Hybrid Systems IEEE Transactions on Automatic Control, Vol.45, No.10, 2000.

[8] Kerrigan, E.C. and Mayne, D.Q. Optimal control of constrained, piecewise affine systems with bounded disturbances. 41st IEEE Conf. on Decision and Control, Las Vegas, Nevada, 10 - 13 December 2002

[9] Bemporad, A. An Efficient Technique for Translating Mixed Logical Dynamical Systems into Piecewise Affine Systems. 41st IEEE Conf. on Decision and Control, Las Vegas, Nevada, December 2002.

[10] Grimble, M. J., and Johnson, M. A., 1988, Optimal multivariable control and estimation theory, Vols. I and II, John Wiley, London.

[11] Atherton D. P., 1982, Nonlinear Control Engineering, Van Nostrand Reinhold, New York.

[12] Isidori, A., 1995, Nonlinear Control Systems, Springer, London.

[13] Hammett, K. D., 1997, Control of non-linear systems via state-feedback state-dependent Riccati equation techniques, $\mathrm{PhD}$ Dissertation, Air Force Institute of Technology, Dayton, Ohio.

[14] Anderson B and Moore J., 1979, Optimal Filtering, Prentice Hall, Englewood Cliffs.

[15] Borrelli F., Bemporad A., Fodor M., and Hrovat D., “An MPC/hybrid system approach to traction control," IEEE Trans. Contr. Systems Technology, vol. 14, no. 3, pp. 541-552, May 2006.

[16] Grimble M J and Pang Y, NGMV control of state dependent multivariable systems, 46th IEEE Conference on Decision and Control, New Orleans, 12-14 Dec. 2007, 1628-1633.

[17] Grimble, M.J. and Majecki, P. Nonlinear GMV control for unstable state-dependent multivariable models, Proceedings of the $47^{\text {th }}$., IEEE Conference on Decision and Control, Cancun, Mexico, Dec. 9-11, 2008. 